\title{
Malignant Cystic Melanoma Metastasis to Brain
}

\begin{abstract}
Metastatic cystic melanoma though rare having a distinctive imaging characteristic due to presence of melanin and hemorrhage. We described a 43-yearsold man presented with reeling of head, short term memory disturbance, ataxia and absence of appetite for one month, gradually developed altered sensorium and vomiting. No past medical history evident. No
\end{abstract}

neurological deficit evident. On primary investigation CT scan shows intra-axial cystic tumor mass over the left temporal region. The patient received total extirpation of the lesion microscopically, followed by chemotherapy and radiotherapy. The pathologic review revealed cystic malignant melanoma metastasis to brain. Patient was doing his normal habit for one month. Suddenly collapse in his home town.

\section{CASE REPORT}

A 43 years old male was admitted to the Neurotrauma Centre of our hospital in September 2014 with the complaint of reeling of head, short term memory disturbance, ataxia and absence of appetite for one month. His symptoms worsened for 10 days and he developed altered sensorium and vomiting because of which he was referred to our hospital for further management from local hospital. He was a known case of hypothyroidism. On examination BP-134/86 mmHg, Pulse62/min, Respiration rate-18/min, HGT-157mg/dl, Chest-B/L VBS, CVS-S1 S2 M0, CNS -drowsy, confused, GCS-E3 V4M5, P/A- Soft, Bowel sound-++ LOS, Pupil-B/L 3mm $R T L$, Plantar-B/L withdrawal.

On first admission, non contrast Computed Tomography (NCCT) was done and demonstrates a cystic lesion in left temporo-parietal region with eccentric hyperdense mural nodule in wall. Small punctuate calcification seen within the nodule. Blood- fluid level were seen within cyst cavity. The lesion has minimal perilesional oedema [Table/Fig-1].

Radiologically differential diagnosis of hemangioblastoma, cystic astrocytoma, ganglioglioma and pleomorphic xanthoastrocytoma were made. Images did not follow signal intensity pattern of normal malignant melanoma, which usually show high signal intensity on T1W1 and low signal intensity on T2W1.

Patient underwent surgery. Osteoplastic craniotomy was done. Dura opened by trans sulcal approach. Large intraaxial
Keywords: Melanocyte, Neural crest cell, Pleomorphic

cystic tumour with extremely vascular mural nodule was seen. Total resection of the tumour was performed. Inside cyst huge brown colour oily fluid present. Post operative hospital stay was uneventful. Patient improved with surgical and medical management.

Histopathological study revealed a highly cellular tumor invading into the white matter. The cells are pleomorphic characterized by round to oval shape, abundant amount of eosinophilic cytoplasm and prominent nucleoli. Several cells are binucleated. Many of the tumor cells contain brown, coarse pigments that fill up cytoplasm are melanin in nature.

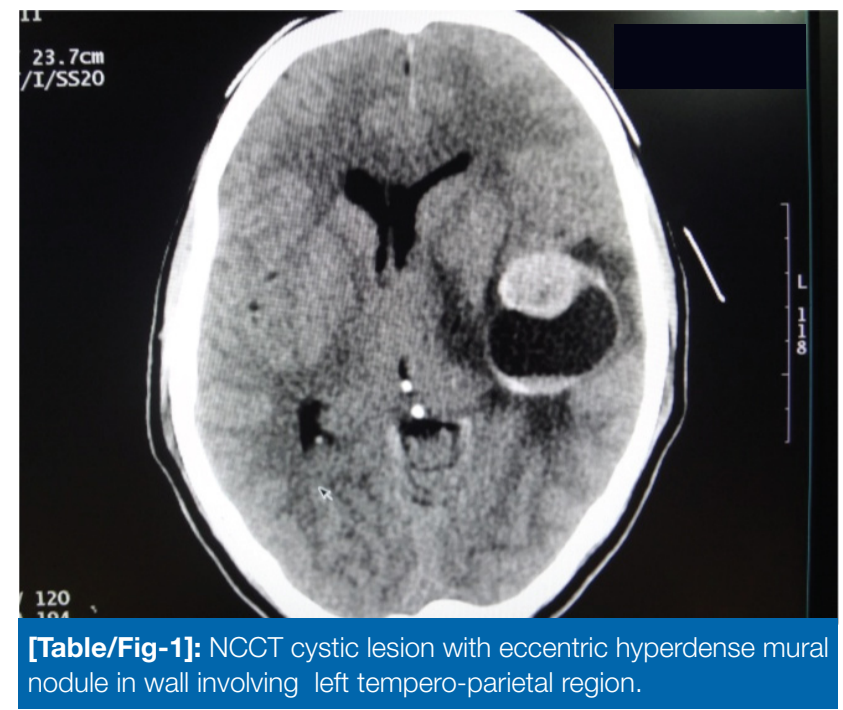


Good numbers of mitotic figures are seen. several foci shows area of hemorrhage and necrosis. The adjoining white matter is edematous and shows reactive gemistocytic astrocytes.

The histological appearance is suggestive of malignant melanoma. Immunohistochemistry could not be done. On retrospective examination two skin nodules, one on thigh region and another on chest wall were found. Biopsy was taken, revealed malignant melanoma. The further examination includes CT-scan of the chest, abdominal, and the pelvic region which were all negative. Patient underwent six cycle of chemotherapy and 10 fractions of radiotherapy. Patient was doing well for one month, but suddenly expired after few days.

\section{DISSCUSSION}

Melanocytes found in skin, mucous membranes, leptomeninges, cerebral parenchyma, and uveal tissue derived from neural crest cells of neuroectoderm during embryogenesis [1-3]. Most melanomas arise in the skin as melanocytes predominantly present in the basal layer of the epidermis. Primary intra cranial melanoma is uncommon, and derived from melanocytes present in leptomeninges. In most instances, melanomas involving the CNS represent metastatic disease. Metastasis tend to occur several years after the primary.

Melanoma cells usually follow different patterns of metastatic spread. Metastasis to the regional lymph nodes most frequently seen with primary melanomas whereas, tumors located in the extremities and trunk region usually shows satellite or in-transit metastasis. In contrast tumor located in the head and neck region metastasize through all the three routes [4]. Cerebral metastasis usually occurs by hematogenous route as it is devoid of lymphatic drainage [5].

Melanoma is the third most common tumor of cerebral metastasis after lung, breast and colon cancers [6, 7]. Though cerebral metastasis occurs only in $10 \%$ of cases but increased to $36-54 \%$ in case of advanced melanoma.

A population based study of 169.444 cancer patients from 1973 to 2001 in Detroit revealed that $7 \%$ of patients diagnosed with melanoma subsequently developed intracranial metastasis $[8,9]$.

The most common symptoms and signs in melanoma with cerebral metastasis are head ache and seizure. Other manifestations include nausea, vomiting, altered sensorium, visual disturbances, progressive neurologic deficit and ataxia. May remain asymptomatic in $10 \%$ of cases. Differential Diagnosis includes anaplastic astrocytoma, thyroid carcinoma, choriocarcinoma and renal cell carcinoma. Histopathologically melanoma divided into melanotic or amelanotic depending on percentage of melanotic cells . Melanotic type when greater than $10 \%$ of melanotic cells and amelanotic when containing less than $10 \%$ melanotic cells on histopathology.

Incidence of multiple brain metastases is more common than solitary lesion. In a case series study of metastatic cerebral melanoma by Ginaldi $S$ et al., found $62 \%$ have multiple lesions and 53\% are bilateral [10].

Supratentorial metastases are more common than infratentorial. Predominantly located at grey -white matter junction with surrounding significant vasogenic oedema. Intratumoural hemorrhage is more common in metastatic Melanoma in comparison to other brain metastases due to high vascularity. Cystic degeneration and necrosis are rarely seen $[10,11]$.

In NECT single or multiple nodules of increased attenuation with enhancement of nodule in post contrast study with variable oedema and intratumoural hemorrhage. Among the neuro imaging techniques contrast enhanced MRI has proven more sensitive than CT.

Isiklar et al., observed that MR findings remains consistent in case of melanotic metastases i.e. Hyperintense on T1W1 and Hypointense on T2W1 [12].

MR signal pattern in melanotic melanoma on T1 -weighted images appears hyperintense, hypo on T2, iso or hyper in relation to cortex on proton density-weighted images as free radicals (like indobe semiquinones and semiquinones) within the melanin pigment are paramagnetic and affect a shortening of $\mathrm{T} 1$ and $\mathrm{T} 2$ relaxation time. Amelanotic melanoma similar to other brain tumours: hypointense or isointense on $\mathrm{T} 1$ and hyperintense or isointense on T2 in relation to cortex [13].

MR imaging characteristic of intra cranial metastatic melanoma depending on types: Melanotic - hyperintense on T1and hypo on $\mathrm{T} 2$ relative to cortex with post contrast enhancement.

Amelanotic- hypo on $\mathrm{T} 1$ and iso to hypointense on $\mathrm{T} 2$ relative to cortex with post contrast enhancement.

Hemorrhagic - signal characteristic dpends on the stage of hemorrhage.

Cystic- usually rare and cystic component usually hypointense on T1W1, hyperintense on T2W1.

Subependyma I - Periventricular nodular or Ribbon like with marked post contrast enhancement.

Perineural/Leptomeningeal - Sugar like coating noted on pial surface or along the cranial nerves (9).

\section{CONCLUSION}

Metastases in malignant melanoma can involve both both intra and extracranial structure. Cerebral metastases most 
commonly occours from head and neck region. Other sites of metastases include muscle, nasopharynx, bone, parotid gland, the meninges, choroid plexus, internal auditory canal, orbit and spinal cord. Cystic melanoma is quite rare. The malignant melanoma metastasis can show distinct appearances but diagnostic difficulty arises due to radiological variability. Prognosis is very poor in case of melanoma metastasis to brain with overall survival of 4-5 months. However recent advances in radiation and systemic therapies has made possible to cure some cases.

\section{REFERENCES}

[1] Greco CS, Soffietti R, Bradac GB, et al. Primitive cerebral melanoma: case report and review of the literature. Surg Neurol. 2001; 55:163-68.

[2] Copeland DD, Sink JD, Seigler HF. Primary intracranial melanoma presenting as a suprasellar tumor. Neurosurgery. 1980;6:54245.

[3] Salpietro FM, Alafaci C, Gervasio O, et al. Primary cervical melanoma with brain metastases. Case report and review of the literature. J Neurosurg. 1998;89:659-66.

[4] Chiarion-Sileni V, Murr R, Pigozzo J. Brain metastases from malignant melanoma. Oncology. 2001;13:170-85. [PubMed]

[5] Soong SJ, Harrison RA, McCarthy WH, Urist MM, Balch $\mathrm{CM}$. Factors affecting survival following local, regional, or distant recurrence from localized melanoma. J Surg Oncol. 1998;67(4):228-33.[PubMed].

[6] McGann GM, Platts A. Computed tomography of cranial metastatic malignant melanoma: features, early detection and unusual cases. Br J Radiol . 1991;64:310-13.

[7] Gupta G, Robertson AG, MacKie RM. Cerebral metastases of cutaneous melanoma. Br J Cancer. 1997;76:256-59

[8] Stone, A, Cooper J, Koenig KL, et al. A comparison of survival rates for treatment of melanoma metastatic to the brain. Cancer Invest. 2004; 22: 492-97.

[9] Barnholtz-Sloan JS, Sloan AE, Davis FG et al. Incidence proportions of brain metastases in patients diagnosed (1973 to 2001) in the Metropolitan Detroit Cancer Surveillance System. $J$ Clin Oncol. 2004;22 (14): 2865-72.

[10] Ginaldi S, Wallace S, Shalen P et al. Cranial computed tomography of malignant melanoma. AJR Am J Roentgenol. 1981;136 (1): 145-49.

[11] Takahashi I, Sugimoto S, Nunomura M et al. A case of cystic metastatic intracranial amelanotic melanoma-analysis of findings in CT and MRI. No To Shinkei. 1991;42 (11): 1031-34. Pubmed citation.

[12] Isiklar I, Leeds NE, Fuller GN, Kumar AJ. Intracranial metastatic melanoma: correlation between MR imaging characteristics and melanin content. AJR Am J Roentgenol.1995; 165 (6): 1503-12.

[13] Cemil B, Emmez H, Oztanir N, Tokgoz N, Dogulu F.Acystic amelanotic melanomametastasis to the brain: case report. Neurocirugia (Astur). 2008; 19(4):365-67.

\section{AUTHOR(S):}

1. Dr. Mamata Singh

2. Dr. Prabhat Nalini Rautray

3. Dr. Lalatendu Swain

\section{PARTICULARS OF CONTRIBUTORS:}

1. Assistant Professor, Department of Radiodiagnosis, S.C.B. Medical College, Cuttack; Visiting Consultant, Neurotrauma Centre, Ashwini Hospital, Cuttack, Odisha, India.

2. Senior Resident, Department of Radiodiagnosis, S.C.B. Medical college, Cuttack, Odisha, India.

3. Associate Professor, Department of Anatomy, S.C.B. Medical College, Cuttack; Visiting Consultant,
Neurotrauma Centre, Ashwini Hospital, Cuttack, Odisha, India.

\section{NAME, ADDRESS, E-MAIL ID OF THE CORRESPONDING AUTHOR:}

Dr. Prabhat Nalini Rautray,

Flat No-112, Metromanorama Complex, Mangalabag, Cuttack-753001, Odisha, India.

E-mail: nalinirautray@gmail.com

FINANCIAL OR OTHER COMPETING INTERESTS: None.

Date of Publishing: Apr 01, 2016 